\title{
Revisiting the Surface Sensitivity of Nanoplasmonic Biosensors
}

\author{
Jiaqi Li, ${ }^{*}, \dagger, \downarrow$ Jian Ye, ${ }^{\dagger, \S}$ Chang Chen, ${ }^{\dagger, \dagger} \mathrm{Yi} \mathrm{Li}^{\dagger, \|}$ Niels Verellen, ${ }^{\dagger, \dagger}$ Victor V. Moshchalkov, \\ Liesbet Lagae, ${ }^{\dagger, \ddagger}$ and Pol Van Dorpe \\ ${ }^{\dagger}$ IMEC, Kapeldreef 75, B-3001 Leuven, Belgium \\ ${ }^{\ddagger}$ INPAC and Department of Physics, KU Leuven, Celestijnenlaan 200 D, B-3001 Leuven, Belgium \\ "Department of Electrical Engineering, KU Leuven, Kasteelpark Arenberg 10, B-3001 Leuven, Belgium
}

\section{Supporting Information}

ABSTRACT: In nanoplasmonic sensing, the bulk refractive index sensitivity is often used as a metric for performance evaluation. However, for biosensing applications, which involve molecular binding events, only the refractive index in a confined region close to the metal surface is altered. The correlation between the bulk and the surface sensitivity strongly depends on the nanostructure geometry, especially in strongly coupled systems. In this paper, we thoroughly investigate the surface sensing performance of diffractively coupled plasmonic crystals using the atomic layer deposition of conformal $\mathrm{Al}_{2} \mathrm{O}_{3}$ layers with well-defined thickness and refractive index. It is demonstrated that the surface sensing capacity cannot be fully described by the bulk sensitivity. It not only shows opposite dependence on the coupling strength compared to the bulk sensitivity, but also the bulk sensitivity cannot reflect the fact that the surface sensitivity could be different in different thickness ranges on the metal surface. The reason rests on the different decay

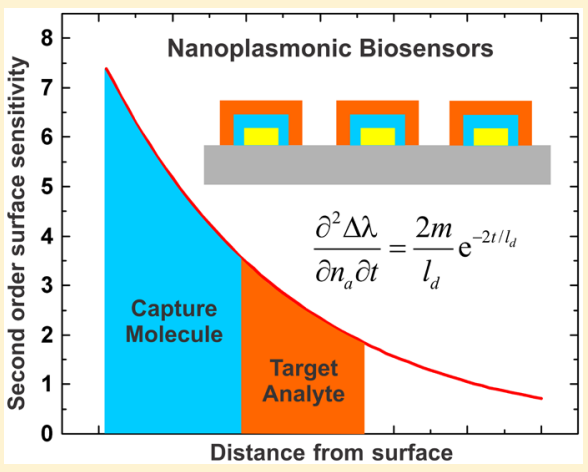
lengths of the plasmonic crystal arrays with different coupling strengths and can be well explained by the second order surface sensitivity that has recently been proposed. Furthermore, we provide a quantitative method to evaluate the surface sensing performance of specific target analyte. This method is generic and can be applied to other nanoplasmonic systems and a broad range of biomolecules with various sizes.

KEYWORDS: surface plasmon resonance, plasmonic crystals, bulk sensitivity, surface sensitivity, decay length

$\mathrm{N}$ anoplasmonic biosensors take advantage of the resonant spectral shifts induced by the molecular binding on the metal nanostructure surface. ${ }^{1,2}$ Due to the miniature sizes, they are promising candidates for high-throughput, multiplexed, and quantitative detections of a broad range of biomolecules. ${ }^{3-6}$ In a common biosensing scheme, the metal nanostructures are functionalized with capture molecules through various surface chemistry modification methods. These capture molecules preferentially recognize the target analyte by specific binding interactions. However, since the electric field of the metal nanostructures is highly confined and decays away from the surface, the capture molecules occupy the most sensitive regions and degrade the sensitivity to the target analyte. ${ }^{7}$ Therefore, how to characterize the sensitivity and apply it for optimum biosensor design is of great significance. ${ }^{8}$

In the literature, the bulk sensitivity is extensively evaluated, which measures the refractive index change to the spatial infinite and covering all the accessible volume of the plasmonic electric field. ${ }^{9-12}$ This bulk sensitivity, divided by the resonance full width at half-maximum (FWHM), is also used in the conventional definition of the figure of merit (FoM) to indicate the general sensor performance. Nevertheless, the bulk sensitivity and thus defined FoM do not take into account the fact that the sensitivity of plasmonic biosensors decays rapidly away from the surface, and their performance also depends on the sizes of the capture molecule and the target analyte. On the contrary, the surface sensitivity, measuring a fraction of the electric field close to the metal surface, is more pertinent to biosensing applications. The bulk and surface sensitivity are related to the plasmonic electric field distributions, and could be fundamentally different. ${ }^{13,14}$ For example, in localized surface plasmons (SPs), the bulk sensitivity is much lower than that of the propagating SPs, although their surface sensitivities have been reported to be comparable. ${ }^{15-17}$ Carefully designed plasmonic nanostructures with a larger bulk sensitivity could also show lower surface signals due to the mismatch of the electric field decay length and the target analyte size. ${ }^{15,18-21}$ We have recently reported that in diffractively coupled plasmonic crystals the bulk and surface sensitivities are even diverging with respect to the FoM. ${ }^{22}$ Therefore, the commonly studied bulk sensitivity is not the optimal parameter to indicate the biosensing performance.

The surface sensitivity has been investigated by using selfassembled monolayers (SAMs) of alkanethiols with various chain lengths, ${ }^{23-26}$ multilayers of oppositely charged polyelectrolytes, ${ }^{15,20,27,28}$ and atomic layer deposition (ALD) of conformal dielectric layers. ${ }^{29-32}$ Although there have been

Received: December 22, 2014 

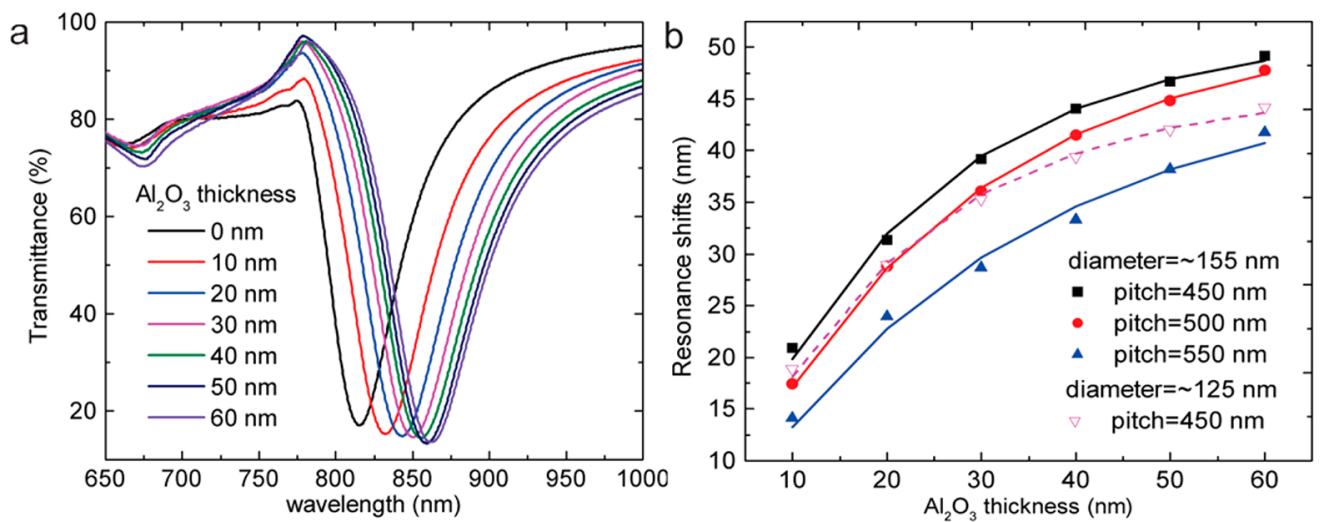

Figure 1. Surface sensitivity measurements using the ALD method. (a) Transmission spectra measured in ethanol for the array with the pitch of 500 $\mathrm{nm}$ and the nanodisk diameter of $\sim 155 \mathrm{~nm}$ for different $\mathrm{Al}_{2} \mathrm{O}_{3}$ layer thicknesses. (b) SLR position shifts as a function of the $\mathrm{Al}_{2} \mathrm{O}_{3}$ thickness. The solid and dashed curves are fitted according to eq 1 .

definitions of surface sensitivity based on the biomolecule size, ${ }^{1,15,33}$ they disregard that the biosensor performance is also affected by the distance of the target analyte away from the metal surface. ${ }^{7}$ A practical and reliable definition of surface sensitivity is still lacking, especially in strongly coupled plasmonic systems, such as Fano resonances. ${ }^{15,34,35}$ We have recommended a second order surface sensitivity in our previous report, ${ }^{22}$ and it was corroborated by the detection of protein bilayers. Nevertheless, the size and refractive index of the proteins are difficult to be precisely characterized. For this reason, how accurate the second order surface sensitivity can describe the surface sensing performance is still an open question. Therefore, in this paper we further investigate the surface sensitivity using the ALD method to deposit conformal dielectric $\mathrm{Al}_{2} \mathrm{O}_{3}$ layers with well-defined thickness and refractive index. To be specific, we thoroughly measure the resonance shifts induced by the surface $\mathrm{Al}_{2} \mathrm{O}_{3}$ layers in the diffractively coupled plasmonic crystals and compare it to the bulk sensitivity as a function of the coupling strength. It is demonstrated that the bulk sensitivity cannot fully describe the surface sensing performance. We quantitatively evaluate the differences between the bulk and the surface sensitivity based on the second order surface sensitivity. In addition, a reliable method is proposed to more accurately assess the resonance shifts caused by the target analyte. This method is generic and can be applied to other nanoplasmonic structures, such as nanorods and nanoholes. ${ }^{3-5}$ It is also useful for a broad range of biomolecules with various sizes, from a thin SAM to bigger viruses.

\section{RESULTS AND DISCUSSION}

Plasmonic crystals are ordered arrays of metal nanostructures and have been well-known to achieve extremely sharp spectral features. $^{36-42}$ By forming a two-dimensional grating system in the periodic array, the diffractive coupling of the localized SP resonance (LSPR) of the metal nanostructures leads to the surface lattice resonance (SLR) mode, which could be considered as a Fano resonance between the broad LSPR and the narrow Rayleigh anomaly (RA) resonance. ${ }^{41}$ The coupling strength of the SLR can be easily adjusted by engineering the nanostructure geometry and the array pitch, and a broad range of FWHMs and sensor FoMs can be achieved. In this investigation, periodic square arrays of $\mathrm{Au}$ nanodisks are also used in order to take advantage of the highly tunable coupling strengths. (SEM images and spectra with controllable FWHMs are shown in Figures $S 1$ and S2, respectively.) If the array pitch is fixed (thus, the RA resonance), larger $\mathrm{Au}$ nanodisks demonstrate broader FWHMs, indicating stronger diffractive coupling. ${ }^{41,42}$ Therefore, in this paper the bulk and surface sensitivities will be studied as a function of the FWHM, which are further categorized for different array pitches.

One of the methods to measure the bulk sensitivity is to immerse the nanoplasmonic structures in liquids with various refractive indexes (Figure S3). All the electric field around the metal nanostructures above the substrate can be accessed and should be taken into account when analyzing the corresponding resonance shifts. On the contrary, only a confined region on the sensor surface changes the refractive index for biomolecule detections. Hence, in order to investigate the surface sensitivity, the ALD method is employed, which is capable of forming conformal $\mathrm{Al}_{2} \mathrm{O}_{3}$ dielectric layers with high uniformity and controllable thickness (Figure $\mathrm{S} 4$ ). The refractive index of the deposited $\mathrm{Al}_{2} \mathrm{O}_{3}$ layers is also well-defined (Figure S5). In Figure 1a, the results of a representative array with the pitch of $500 \mathrm{~nm}$ and the $\mathrm{Au}$ nanodisk diameter of $\sim 155 \mathrm{~nm}$ are shown. The spectra were measured in ethanol instead of index matching oils so that the $\mathrm{Al}_{2} \mathrm{O}_{3}$ surface chemistry can be preserved for the sequential depositions. Nevertheless, the tunability of the coupling strength is maintained in ethanol for the nanodisk sizes under our consideration (Figure S2c,d).

It is demonstrated in Figure 1a that the SLR dips red shift as the $\mathrm{Al}_{2} \mathrm{O}_{3}$ thickness increases. The spectral shifts per $10 \mathrm{~nm}$ of $\mathrm{Al}_{2} \mathrm{O}_{3}$ also gradually decrease upon the sequential $\mathrm{Al}_{2} \mathrm{O}_{3}$ depositions, implying that the surface sensitivity is dropping. This is because the electric field of the Au nanodisk decays away from the surface. To compare the surface sensitivity of arrays with various coupling strengths, four examples of the spectral shifts as a function of the $\mathrm{Al}_{2} \mathrm{O}_{3}$ thickness are shown in Figure $1 \mathrm{~b}$. For similar nanodisk sizes (solid symbols), the array with smaller pitch shows a higher surface sensitivity. In contrast, arrays with larger pitches demonstrate higher bulk sensitivities (Figure S3b). Similar opposite dependence of the surface and bulk sensitivity on the array pitch has been reported. ${ }^{22}$ It is also observed that the spectral shift curves against the $\mathrm{Al}_{2} \mathrm{O}_{3}$ thickness could intersect (open purple vs solid red symbols), suggesting that over different thickness ranges the surface sensitivity can be different. FDTD simulations for similar arrays corroborate the results (Figure S6). Therefore, it is evident that 

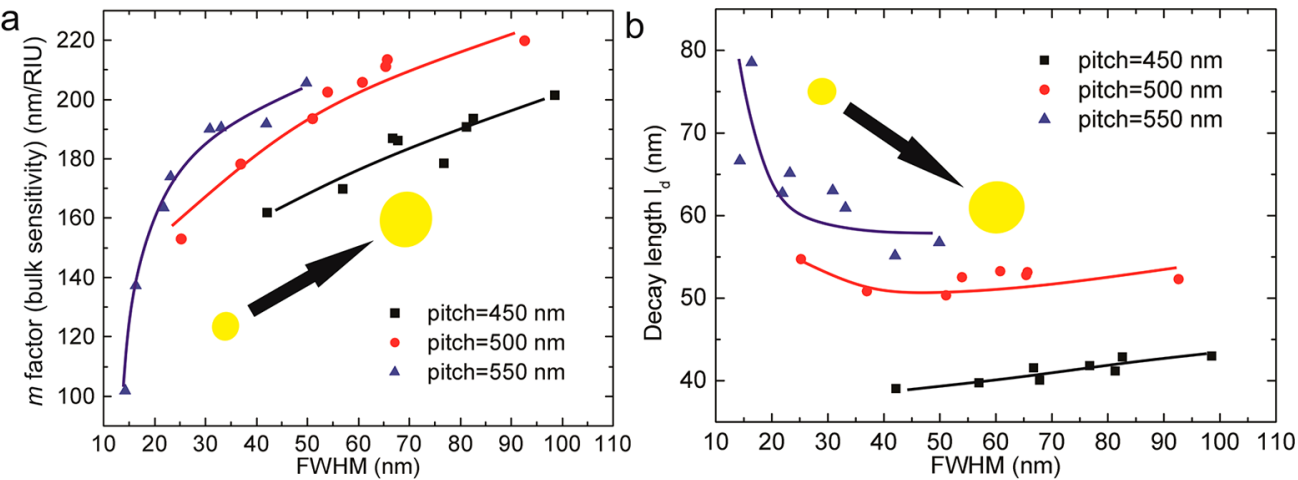

Figure 2. Experimental results of (a) $m$ sensitivity factor (bulk sensitivity) and (b) effective decay length $l_{\mathrm{d}}$ obtained by fitting to eq 1 as a function of the FWHM. The FWHMs are measured for arrays without the $\mathrm{Al}_{2} \mathrm{O}_{3}$ depositions. The solid lines are to guide the eyes. The black arrows indicate the increasing trend of the Au nanodisk diameters for the data set of each array pitch.

the surface sensing performance cannot be adequately described with only the bulk sensitivity.

The surface sensitivity strongly depends on the electric field distributions. Since the field decays away from the metal surface, the surface sensitivity should be a function of the distance from the surface. Hence, the bulk sensitivity, measuring the whole accessible volume of the electric field, is too oversimplified to be a benchmarking metric. Moreover, the common biosensing scheme with the capture molecules pushing away target analyte from the surface makes it a more complicated problem. A practical definition of surface sensitivity better takes into account the decay of the electric field. It should consider the distance of the target analyte away from the metal surface and be able to estimate the spectral shifts of biomolecules with various sizes. It should also acknowledge the role of refractive index as in the definition of the bulk sensitivity.

Further analysis of the surface sensitivity is based on the theoretical equation considering both the adsorbate index $n_{\text {adsorbate }}$ and thickness $t^{43}$

$$
\Delta \lambda=m \times\left(n_{\text {adsorbate }}-n_{\text {medium }}\right) \times\left(1-e^{-2 t / l_{\mathrm{d}}}\right)
$$

where $m$ is the sensitivity factor, $n_{\text {medium }}$ is the refractive index of the medium, and $l_{\mathrm{d}}$ is the decay length of the electric field. Equation 1 was first proposed for propagating SPs to estimate the adsorbate thickness and surface coverage and later used to interpret surface sensing performance of localized SPs. ${ }^{25,26,28,30,44}$ This equation takes a simple one-dimensional exponential decay length and is sufficient to describe the propagating SPs on a planar metal surface. On the contrary, the localized SPs of asymmetrically shaped nanostructures are a 3D problem. However, specific to the biosensing scheme, the capture molecules can be modeled as a conformal layer on the metal nanostructure surface, and the estimate of the spectral shifts of conformal layers can also be used for the target analytes considering their random adsorption locations on the surface. This simplifies the $3 \mathrm{D}$ problem into a $1 \mathrm{D}$ case, and the decay length in eq 1 can be considered as an effective quantity specific to such a biosensing scheme. It is also consistent with the ALD method used in our paper. In addition, in cases where the full coverage assumption is not possible, ${ }^{45,46}$ the following surface sensitivity analysis method can be extended as well by forming conformal layers on specific locations.

Using eq 1, we have fitted the spectral shifts against the $\mathrm{Al}_{2} \mathrm{O}_{3}$ layer thicknesses, and the obtained curves are shown in Figures $\mathrm{lb}$ and S6. Through such fittings, the sensitivity factor $m$ and the decay length $l_{\mathrm{d}}$ could be extracted, and the results are summarized in Figure 2 (similar FDTD simulation results are available in Figure S7).

In eq 1 , as the adsorbate thickness $t$ approaches to infinite, the sensitivity factor $m$ should be the bulk sensitivity. We have compared the $m$ sensitivity factors obtained by this method to the bulk sensitivities experimentally measured using aqueous glycerol solutions over a refractive index range close to $n_{\text {ethanol }}=$ 1.36 (Figure S8), and these two quantities are similar to each other. This confirms that the $m$ factor equals the bulk sensitivity in eq 1. Figure 2 a demonstrates the bulk sensitivity as a function of the FWHM. The $450 \mathrm{~nm}$ pitch array shows lower bulk sensitivity, contrasting the surface sensitivity results in Figure $1 \mathrm{~b}$ as well. Notably, in arrays with narrower FWHMs (less than $30 \mathrm{~nm}$ ), the bulk sensitivity drops fast with reducing FWHM. In these arrays, the electric field is less confined on the nanodisk surface (larger decay length $l_{\mathrm{d}}$, as discussed below). Due to the index mismatch between the ethanol and the quartz, the field is more concentrated in the quartz substrate, becoming inaccessible to the background refractive index change. Therefore, the bulk sensitivity is greatly decreased.

The obtained effective decay length $l_{\mathrm{d}}$ is plotted in Figure $2 \mathrm{~b}$. For the $450 \mathrm{~nm}$ pitch array, the decay length slightly increases with the nanodisk size, since the sensing volume increases with the size as well. For the arrays with narrow FWHMs (less than $30 \mathrm{~nm}$ ), the $l_{\mathrm{d}}$ increases abruptly with reducing nanodisk sizes due to the diffractive coupling between the nanodisks, implying that the electric field is more extended into the medium. It can also provide an explanation for the opposite dependence of the bulk and surface sensitivity on the array pitch. The bulk sensitivity is determined by the entire medium volume above the substrate surface, while the surface sensitivity only probes a fraction of that volume. The fraction occupied by the adsorbate dictates the corresponding spectral shifts. ${ }^{47}$ For a larger pitch array, although the bulk sensitivity is higher, its larger decay length $l_{\mathrm{d}}$ gives rise to the lower electric field fraction occupied by the adsorbate, resulting in the smaller surface sensitivity.

Based on these two quantities, $m$ sensitivity factor and decay length $l_{\mathrm{d}}$, the second order surface sensitivity can be calculated as the second order derivative of the spectral shift $\Delta \lambda$ in eq 1 :

$$
\frac{\partial^{2} \Delta \lambda}{\partial n_{\mathrm{a}} \partial t}=\frac{2 m}{l_{\mathrm{d}}} e^{-2 t / l_{\mathrm{d}}}
$$

This definition takes into account the surface sensitivity dependence on both the refractive index and the size of the 
adsorbate. It reflects that the sensitivity relates to the distance of the target analyte away from the surface, indicated by $t$. It is also evident that the sensitivity decays away from the nanostructure surface, and the decaying rate can be characterized by the exponential decay length $l_{\mathrm{d}}$. This surface sensitivity also directly correlates to the bulk sensitivity ( $m$ factor).

An essential factor in this surface sensitivity is the prefactor $2 m / l_{\text {d }}$, which can be considered as the sensitivity when the adsorbate thickness $t$ approaches zero or the sensitivity very close to the surface. Since it is a quantity with $l_{\mathrm{d}}$ as the denominator, it indicates that a large decay length can result in a small sensitivity at the nanostructure surface. In Figure 3, the

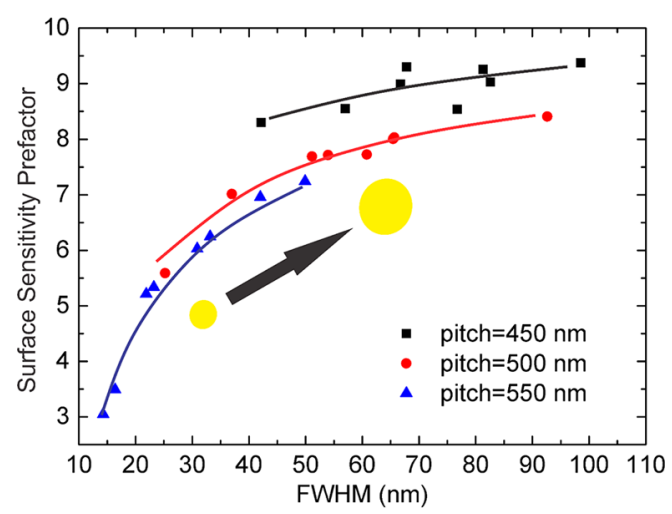

Figure 3. Experimental results of the second order surface sensitivity prefactor $2 \mathrm{~m} / \mathrm{l}_{\mathrm{d}}$ as a function of the FWHM. The FWHMs are measured in ethanol for arrays without the $\mathrm{Al}_{2} \mathrm{O}_{3}$ depositions. The solid lines are to guide the eyes. The black arrow indicates the increasing trend of the Au nanodisk diameters for the data set of each array pitch.

dependence of this prefactor $2 m / l_{\mathrm{d}}$ on the FWHM is demonstrated (FDTD simulation results are available in Figure S9). The $450 \mathrm{~nm}$ pitch array shows the largest $2 \mathrm{~m} / \mathrm{l}_{\mathrm{d}}$ since the electric field is mostly confined at the surface, while it rapidly drops down for other arrays with narrower FWHMs due to the largely stretched out electric field.

On top of the discussions above, optimized plasmonic biosensor design needs to carefully evaluate the second order surface sensitivity, namely the prefactor $2 \mathrm{~m} / \mathrm{l}_{\mathrm{d}}$ and the decay length $l_{\mathrm{d}}$. With these two quantities, more reliable insight on the biosensor performance can be gained. The surface sensitivity is largest at the very surface, indicated by the prefactor $2 \mathrm{~m} / l_{\mathrm{d}}$, and exponentially decays away with a rate defined by $l_{\mathrm{d}}$. The bulk sensitivity or $m$ factor cannot solely determine the surface sensing performance since the effect of the decay length $l_{\mathrm{d}}$ is quite significant. The ultimate goal is to design a biosensor with a prefactor $2 \mathrm{~m} / l_{\mathrm{d}}$ as large as possible and a decay length $l_{\mathrm{d}}$ that matches the target biomolecule size. However, the close correlation between the prefactor $2 \mathrm{~m} / \mathrm{l}_{\mathrm{d}}$ and the decay length $l_{\mathrm{d}}$ implies that careful assessment is needed.

In Figure 4a, one example of the second order surface sensitivity is plotted against the distance from the surface, and the curve is applied for the common biosensing scheme. It shows that the sensitivity decays away from the surface. The area below the curve, which is the integral over the thickness, indicates the sensitivity to the biomolecule refractive index. The capture molecule pushes the target analyte a distance of $t_{\mathrm{C}}$ away from the biosensor surface, and exponentially degrades the sensitivity to the target by a factor of $\exp \left(-2 t_{\mathrm{C}} / l_{\mathrm{d}}\right)$. The area corresponding to the thickness range of the target analyte $\left(t_{\mathrm{C}}\right.$ to $t_{\mathrm{C}}+t_{\mathrm{T}}$ in Figure $4 \mathrm{a}$ ) provides a more accurate estimate of the resonance shifts to the specific target. This graphic method is equal as integrating eq 2 in the corresponding thickness range. Therefore, the sensitivity performance of a nanoplasmonic biosensor can be simply evaluated by the area in the thickness range specific to the target biomolecule.

In addition, as shown in Figure $4 b$, because of the differences in the surface sensitivity prefactor $2 \mathrm{~m} / l_{d}$ and the decay length $l_{\mathrm{d}}$ the sensitivity curves can intersect, meaning that over different thickness ranges the sensitivity can be different. The shadowed areas in black/red colors indicate the range in which the corresponding array is more sensitive. To more accurately evaluate the biosensing performance, the thickness range of the target should be identified first. If the target analyte is located in the black region, the array corresponding to the black curve is more sensitive; if in the red region, the other array is recommended. Therefore, compared to the commonly used bulk sensitivity, the second order surface sensitivity offers a more intuitive and reliable method to estimate the biosensing performance as well as compare the sensitivity to different types of target biomolecules. As a rule of thumb, if both the capture molecule and target analyte are small in size and close to the
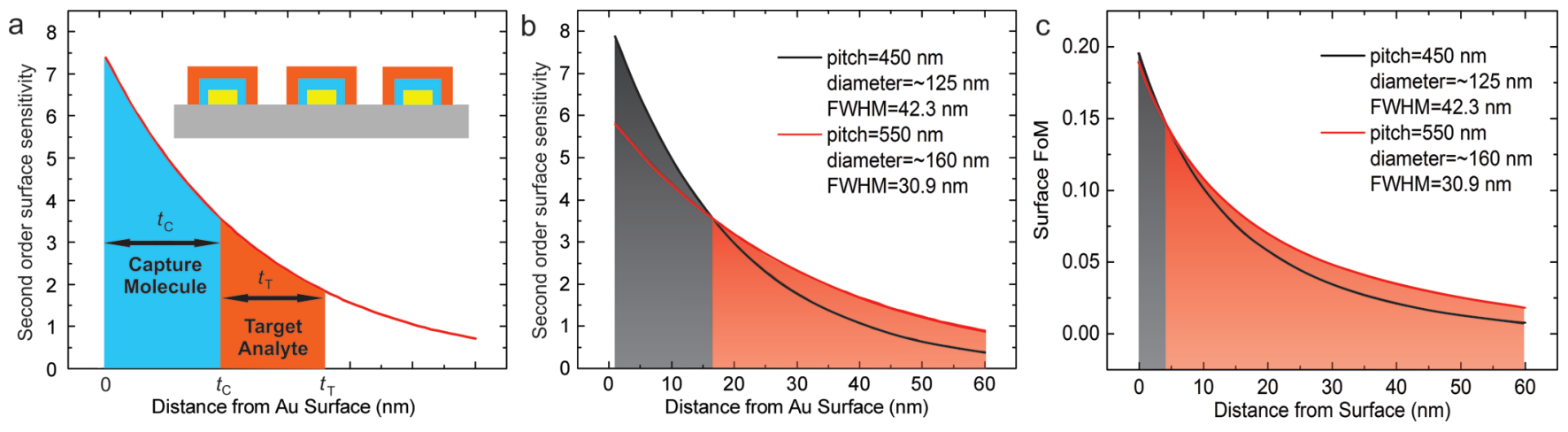

Figure 4. (a) Second order surface sensitivity applied for the common biosensing scheme. The sizes of the capture molecule and target analyte are indicated as $t_{\mathrm{C}}$ and $t_{\mathrm{T}}$, respectively. The blue/orange areas indicate the sensitivity to the refractive index change in the thickness range specific to the capture molecule/target analyte. Comparisons of (b) the second order surface sensitivity curve and (c) surface FoM for two arrays with different coupling strengths. The shadowed areas show the thickness ranges in which the curves in corresponding colors have the higher surface sensitivity or surface FoM. 

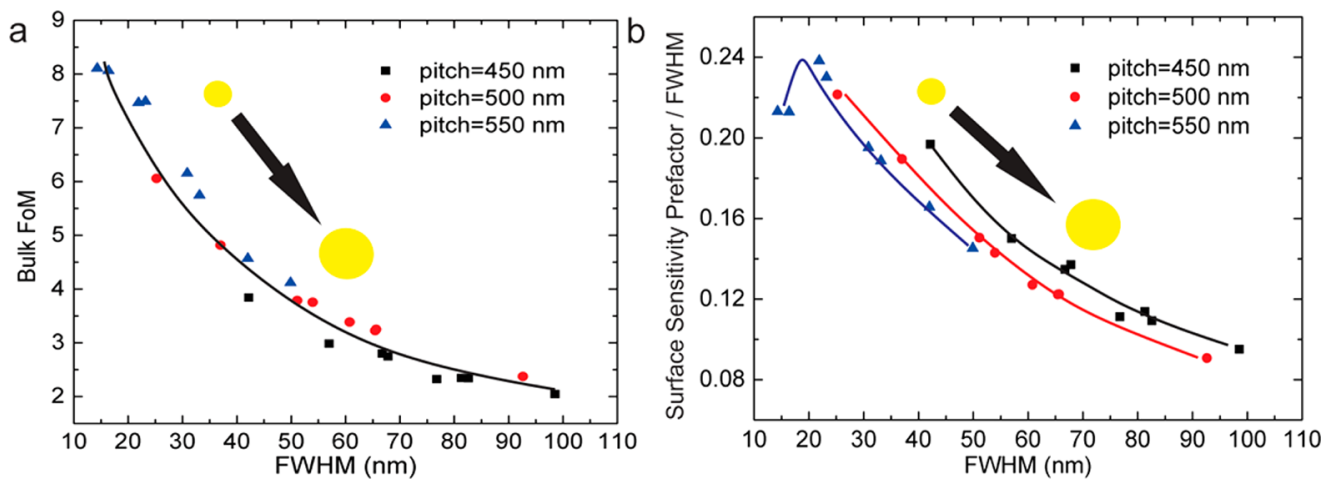

Figure 5. Experimental comparisons between (a) the FoM defined by the bulk sensitivity and (b) the FoM defined by the surface sensitivity prefactor $2 \mathrm{~m} / \mathrm{l}_{\mathrm{d}}$ as a function of the FWHM. The solid lines are to guide the eyes. The black arrows indicate the increasing trend of the Au nanodisk diameters for the data set of each array pitch.

metal surface (e.g., a thin SAM in direct contact with the surface is the target without capture molecules), an array with a small pitch should be used to take advantage of its highly confined surface electric field, even though its bulk sensitivity is small. In contrast, if a larger molecule (e.g., a virus of tens of nanometers in size or bigger) is the target, or the capture molecule is large, pushing the target analyte far away from the surface, a larger pitch array with an extended electric field distribution is a better choice. In this manner, the diffractively coupled plasmonic crystals provide a flexible platform with easily tunable coupling strength to better match different types of target analyte in various biosensing schemes.

Moreover, in plasmonic sensing applications, the spectral resolution, indicated by the FWHM, is the other important performance factor. A narrower FWHM can result in higher spectral resolution. ${ }^{35,48,49}$ Conventionally, the FoM is defined as the bulk sensitivity over the FWHM (named bulk FoM here). It has been reported that a universal scaling rule of the bulk FoM is present for the diffractively coupled plasmonic crystals. ${ }^{41}$ It entails that the spectral difference between the SLR and the RA alone can define the bulk FoM. In Figure 5a, the bulk FoM as a function of the FWHM is plotted, and it demonstrates that the largest bulk FoM appears in the array with the narrowest FWHM. Nevertheless, the case is more complex in biosensing applications. Its performance depends on the specific biosensing scheme and the sizes of the capture molecule and target analyte. Based on the second order surface sensitivity, a surface FoM can be defined in eq 3.

$$
\text { FoM } \text { surface }_{\text {s. }}=\frac{\frac{2 m}{l_{\mathrm{d}}} \exp \left(-\frac{2 t}{l_{\mathrm{d}}}\right)}{\text { FWHM }}
$$

It can be evaluated using the similar method as for the surface sensitivity discussed above. Figure $4 \mathrm{c}$ demonstrates the surface FoM of the two arrays in Figure $4 \mathrm{~b}$. The dependence of the FWHM on the distance $t$ has been taken into account by fitting the experimental data using a polynomial function. The two curves could intersect as well, indicating different surface FoM values in different thickness ranges. The intersection position is closer to the surface since the smaller FWHM of the array with the $550 \mathrm{~nm}$ pitch makes the difference of the surface sensitivity less significant.

A FoM specific to the target can also be defined as

$$
\begin{gathered}
\text { FoM }_{\text {target }}=\frac{\int_{t_{\mathrm{C}}}^{t_{\mathrm{T}}+t_{\mathrm{C}}} \frac{2 m}{l_{\mathrm{d}}} \exp \left(-\frac{2 t}{l_{\mathrm{d}}}\right) \mathrm{d} t}{\text { FWHM }} \\
=\frac{m \exp \left(-\frac{2 t_{\mathrm{C}}}{l_{\mathrm{d}}}\right)\left[1-\exp \left(-\frac{2 t_{\mathrm{T}}}{l_{\mathrm{d}}}\right)\right]}{\text { FWHM }}
\end{gathered}
$$

in which the numerator is just the area below the second order surface sensitivity curve bounded by the target. The FoM defined in this manner can be more instructive for nanoplasmonic biosensor design, and thorough assessment on a case-by-case basis is needed. As an example, we take the prefactor $2 m / l_{\mathrm{d}}$ of the second order surface sensitivity as the numerator in the FoM, and plot it against the FWHM as shown in Figure $5 \mathrm{~b}$. This $2 m / l_{\mathrm{d}}$ over FWHM can reflect the performance of a thin SAM detection. Contrast to the bulk FoM, in the array with the narrowest FWHM, the SAM FoM drops down due to the larger decay length $l_{\mathrm{d}}$ and is not the highest any more. Supporting FDTD simulated results are available in Figure S10.

\section{CONCLUSION}

In summary, we have investigated the surface sensing performance in diffractively coupled plasmonic crystals composed of periodic $\mathrm{Au}$ nanodisks using the ALD method. By sequentially depositing conformal $\mathrm{Al}_{2} \mathrm{O}_{3}$ dielectric layers with different thicknesses, the refractive index in a confined region on the plasmonic crystal array surface can be accurately modified. The surface sensitivity shows an opposite dependence on the array pitch compared to the bulk sensitivity and can also be different over different thickness ranges away from the nanodisk surface. The second order surface sensitivity not only well explains the surface sensing performance, but also provides a more reliable method for nanoplasmonic biosensor design. The area below the second order surface sensitivity curve in the thickness range specific to the target analyte can more precisely estimate the sensitivity and is recommended for the biosensor performance evaluation. The method suggested here is generic and can find broad applications to other nanoplasmonic biosensor systems and biomolecules with various sizes. It can be interesting to apply this method to investigate the surface sensing performance of nanoplasmonic biosensors with various other coupling strategies, such as coupling to the Fabry-Perot mode or propagating SP. It is also not limited by the biomolecule size under consideration so that a diversity of sensing applications can be evaluated. Matching 
the target biomolecule size with the optimum coupling strategy will maximize the nanoplasmonic biosensing potential.

\section{METHODS}

The periodic $\mathrm{Au}$ nanodisk square arrays were fabricated on quartz substrates by electron beam lithography using negative resist ma-N2400.6 (micro resist technology GmbH). The array size is $500 \mu \mathrm{m} \times 500 \mu \mathrm{m}$. The nanodisk height is kept a constant at $30 \mathrm{~nm}$. A Philips XL30 FEG instrument operated at an accelerating voltage of $5 \mathrm{kV}$ was used to take the SEM images. The transmission spectra were measured with a Bruker Vertex 80v Fourier transform infrared (FTIR) spectrometer equipped on a Hyperion 2000 microscope using collimated excitation light. The bulk sensitivity was measured using either refractive index matching oils (Cargille Laboratories, from $n=$ $1.52-1.58)$, or aqueous glycerol solutions with a series of refractive indexes (from $n=1.333-1.4011$ ). Liquid was sequentially injected into a custom-made flow cell starting from low to high refractive index. The surface index change was realized by $\mathrm{ALD}$ of conformal $\mathrm{Al}_{2} \mathrm{O}_{3}$ layers up to the thickness of $60 \mathrm{~nm}$ in steps of $10 \mathrm{~nm}$ (ALD Savannah). Before the first ALD step, the Au nanodisk surface was functionalized with 1 $\mathrm{mM}$ ethanol solution of 16-mercaptohexadecanoic acid overnight in order to make the Au surface compatible with the following ALD process, ${ }^{50}$ rinsed thoroughly with ethanol, and dried with $\mathrm{N}_{2}$ gas. The transmission spectra after each ALD step were taken in ethanol to maintain the surface chemistry for the following depositions. The refractive index of the deposited $\mathrm{Al}_{2} \mathrm{O}_{3}$ was characterized using a Sopralab GES5E variable angle spectroscopic ellipsometer. The numerical simulations were run using Lumerical FDTD Solutions v8.5 (Lumerical Solutions Inc.). In the $x$ - and $y$-directions, a periodic boundary condition (BC) with various pitches was taken, while in the $z$-direction perfectly matched layer BC was used. An $x$-polarized plane wave was employed as the incidence light. A mesh size of $2 \mathrm{~nm}$ was taken. The permittivity data of the $\mathrm{Al}_{2} \mathrm{O}_{3}$ was experimentally measured, while the data of the quartz and $\mathrm{Au}$ were taken from the Palik and Chris Johnson handbooks, respectively.

\section{ASSOCIATED CONTENT}

\section{S Supporting Information}

SEM images of plasmonic crystal arrays, transmission spectra, refractive index of $\mathrm{Al}_{2} \mathrm{O}_{3}$ layer, FDTD simulation results, and comparisons between the bulk sensitivity and $m$ factor. This material is available free of charge via the Internet at http:// pubs.acs.org.

\section{AUTHOR INFORMATION}

\section{Corresponding Author}

*E-mail: lijackie@imec.be. Tel.: +32-1628-3567.

\section{Present Address}

${ }^{\S}$ School of Biomedical Engineering and Med-X Research Institute, Shanghai Jiao Tong University, 1954 Huashan Road, Shanghai 200030, China.

\section{Notes}

The authors declare no competing financial interest.

\section{ACKNOWLEDGMENTS}

C.C. and N.V. gratefully acknowledge the financial support from FWO (Flanders). N.V. and V.V.M. are supported by the
Methusalem funding by the Flemish Government. We thank Eric Vancoille for the help with the cross-section SEM images.

\section{REFERENCES}

(1) Mayer, K. M.; Hafner, J. H. Localized Surface Plasmon Resonance Sensors. Chem. Rev. 2011, 111, 3828-3857.

(2) Homola, J. Surface Plasmon Resonance Sensors for Detection of Chemical and Biological Species. Chem. Rev. 2008, 108, 462-493.

(3) Ruemmele, J. a; Hall, W. P.; Ruvuna, L. K.; Van Duyne, R. P. A Localized Surface Plasmon Resonance Imaging Instrument for Multiplexed Biosensing. Anal. Chem. 2013, 85, 4560-4566.

(4) Aćimović, S. S.; Ortega, M. A.; Sanz, V.; Berthelot, J.; GarciaCordero, J. L.; Renger, J.; Maerkl, S. J.; Kreuzer, M. P.; Quidant, R. LSPR Chip for Parallel, Rapid, and Sensitive Detection of Cancer Markers in Serum. Nano Lett. 2014, 14, 2636-2641.

(5) Im, H.; Shao, H.; Park, Y. Il; Peterson, V. M.; Castro, C. M.; Weissleder, R.; Lee, H. Label-Free Detection and Molecular Profiling of Exosomes with a Nano-Plasmonic Sensor. Nat. Biotechnol. 2014, 32, 490-495.

(6) Lee, S. H.; Lindquist, N. C.; Wittenberg, N. J.; Jordan, L. R.; Oh, S.-H. Real-Time Full-Spectral Imaging and Affinity Measurements from 50 Microfluidic Channels Using Nanohole Surface Plasmon Resonance. Lab Chip 2012, 12, 3882-3890.

(7) Feuz, L.; Jonsson, M. P.; Höök, F. Material-Selective Surface Chemistry for Nanoplasmonic Sensors: Optimizing Sensitivity and Controlling Binding to Local Hot Spots. Nano Lett. 2012, 12, 873879.

(8) Piliarik, M.; Kvasnička, P.; Galler, N.; Krenn, J. R.; Homola, J. Local Refractive Index Sensitivity of Plasmonic Nanoparticles. Opt. Express 2011, 19, 9213-9220.

(9) Ye, J.; Van Dorpe, P. Improvement of Figure of Merit for Gold Nanobar Array Plasmonic Sensors. Plasmonics 2011, 6, 665-671.

(10) Verellen, N.; Van Dorpe, P.; Huang, C.; Lodewijks, K.; Vandenbosch, G. A. E.; Lagae, L.; Moshchalkov, V. V. Plasmon Line Shaping Using Nanocrosses for High Sensitivity Localized Surface Plasmon Resonance Sensing. Nano Lett. 2011, 11, 391-397.

(11) Liu, N.; Weiss, T.; Mesch, M.; Langguth, L.; Eigenthaler, U.; Hirscher, M.; Sönnichsen, C.; Giessen, H. Planar Metamaterial Analogue of Electromagnetically Induced Transparency for Plasmonic Sensing. Nano Lett. 2010, 10, 1103-1107.

(12) Lassiter, J. B.; Sobhani, H.; Fan, J. a; Kundu, J.; Capasso, F.; Nordlander, P.; Halas, N. J. Fano Resonances in Plasmonic Nanoclusters: Geometrical and Chemical Tunability. Nano Lett. 2010, 10, 3184-3189.

(13) Estevez, M.-C.; Otte, M. a; Sepulveda, B.; Lechuga, L. M. Trends and Challenges of Refractometric Nanoplasmonic Biosensors: A Review. Anal. Chim. Acta 2014, 806, 55-73.

(14) Anker, J. N.; Hall, W. P.; Lyandres, O.; Shah, N. C.; Zhao, J.; Van Duyne, R. P. Biosensing with Plasmonic Nanosensors. Nat. Mater. 2008, 7, 442-453.

(15) Otte, M. a; Sepúlveda, B.; Ni, W.; Juste, J. P.; Liz-Marzán, L. M.; Lechuga, L. M. Identification of the Optimal Spectral Region for Plasmonic and Nanoplasmonic Sensing. ACS Nano 2010, 4, 349-357.

(16) Zalyubovskiy, S. J.; Bogdanova, M.; Deinega, A.; Lozovik, Y.; Pris, A. D.; An, K. H.; Hall, W. P.; Potyrailo, R. a. Theoretical Limit of Localized Surface Plasmon Resonance Sensitivity to Local Refractive Index Change and Its Comparison to Conventional Surface Plasmon Resonance Sensor. J. Opt. Soc. Am. A 2012, 29, 994-1002.

(17) Svedendahl, M.; Chen, S.; Dmitriev, A.; Käll, M. Refractometric Sensing Using Propagating versus Localized Surface Plasmons: A Direct Comparison. Nano Lett. 2009, 9, 4428-4433.

(18) Barbora, Š.; Homola, J. Sensing Properties of Lattice Resonances of 2D Metal Nanoparticle Arrays: An Analytical Model. Opt. Express 2013, 21, 27490-27502.

(19) Bellapadrona, G.; Tesler, A. B.; Grünstein, D.; Hossain, L. H.; Kikkeri, R.; Seeberger, P. H.; Vaskevich, A.; Rubinstein, I. Optimization of Localized Surface Plasmon Resonance Transducers for Studying Carbohydrate-Protein Interactions. Anal. Chem. 2012, 84, $232-240$. 
(20) Kedem, O.; Tesler, A. B.; Vaskevich, A.; Rubinstein, I. Sensitivity and Optimization of Localized Surface Plasmon Resonance Transducers. ACS Nano 2011, 5, 748-760.

(21) Joshi, G. K.; McClory, P. J.; Muhoberac, B. B.; Kumbhar, A.; Smith, K. A.; Sardar, R. Designing Efficient Localized Surface Plasmon Resonance-Based Sensing Platforms: Optimization of Sensor Response by Controlling the Edge Length of Gold Nanoprisms. J. Phys. Chem. C 2012, 116, 20990-21000.

(22) Li, J.; Ye, J.; Chen, C.; Hermans, L.; Verellen, N.; Ryken, J.; Jans, H.; Van Roy, W.; Moshchalkov, V. V.; Lagae, L.; Van Dorpe, P. Biosensing Using Diffractively Coupled Plasmonic Crystals: The Figure of Merit Revisited. Adv. Opt. Mater. 2014, 3, 176-181.

(23) Larsson, E. M.; Alegret, J.; Käll, M.; Sutherland, D. S. Sensing Characteristics of NIR Localized Surface Plasmon Resonances in Gold Nanorings for Application as Ultrasensitive Biosensors. Nano Lett. 2007, 7, 1256-1263.

(24) Haes, A. J.; Zou, S.; Schatz, G. C.; Van Duyne, R. P. Nanoscale Optical Biosensor: Short Range Distance Dependence of the Localized Surface Plasmon Resonance of Noble Metal Nanoparticles. J. Phys. Chem. B 2004, 108, 6961-6968.

(25) Rindzevicius, T.; Alaverdyan, Y.; Dahlin, A.; Höök, F.; Sutherland, D. S.; Käll, M. Plasmonic Sensing Characteristics of Single Nanometric Holes. Nano Lett. 2005, 5, 2335-2339.

(26) McFarland, A. D.; Van Duyne, R. P. Single Silver Nanoparticles as Real-Time Optical Sensors with Zeptomole Sensitivity. Nano Lett. 2003, 3, 1057-1062.

(27) Nusz, G. J.; Curry, A. C.; Marinakos, S. M.; Wax, A.; Chilkoti, A. Rational Selection of Gold Nanorod Geometry for Label-Free Plasmonic Biosensors. ACS Nano 2009, 3, 795-806.

(28) Haes, A. J.; Zou, S.; Schatz, G. C.; Van Duyne, R. P. A Nanoscale Optical Biosensor: The Long Range Distance Dependence of the Localized Surface Plasmon Resonance of Noble Metal Nanoparticles. J. Phys. Chem. B 2004, 108, 109-116.

(29) Yu, X.; Shi, L.; Han, D.; Zi, J.; Braun, P. V. High Quality Factor Metallodielectric Hybrid Plasmonic-Photonic Crystals. Adv. Funct. Mater. 2010, 20, 1910-1916.

(30) Chen, S.; Svedendahl, M.; Käll, M.; Gunnarsson, L.; Dmitriev, a. Ultrahigh Sensitivity Made Simple: Nanoplasmonic Label-Free Biosensing with an Extremely Low Limit-of-Detection for Bacterial and Cancer Diagnostics. Nanotechnology 2009, 20, 434015.

(31) Savoia, S.; Ricciardi, A.; Crescitelli, A. Surface Sensitivity of Rayleigh Anomalies in Metallic Nanogratings. Opt. Express 2013, 21, 23531-23542.

(32) Whitney, A. V.; Elam, J. W.; Zou, S.; Zinovev, A. V.; Stair, P. C.; Schatz, G. C.; Van Duyne, R. P. Localized Surface Plasmon Resonance Nanosensor: A High-Resolution Distance-Dependence Study Using Atomic Layer Deposition. J. Phys. Chem. B 2005, 109, 20522-20528. (33) Becker, J.; Trügler, A.; Jakab, A.; Hohenester, U.; Sönnichsen, C. The Optimal Aspect Ratio of Gold Nanorods for Plasmonic BioSensing. Plasmonics 2010, 5, 161-167.

(34) Yanik, A. a; Cetin, A. E.; Huang, M.; Artar, A.; Mousavi, S. H.; Khanikaev, A.; Connor, J. H.; Shvets, G.; Altug, H. Seeing Protein Monolayers with Naked Eye through Plasmonic Fano Resonances. Proc. Natl. Acad. Sci. U.S.A. 2011, 108, 11784-11789.

(35) Shen, Y.; Zhou, J.; Liu, T.; Tao, Y.; Jiang, R.; Liu, M.; Xiao, G.; Zhu, J.; Zhou, Z.-K.; Wang, X.; Jin, C.; Wang, J. Plasmonic Gold Mushroom Arrays with Refractive Index Sensing Figures of Merit Approaching the Theoretical Limit. Nat. Commun. 2013, 4, 2381.

(36) Chu, Y.; Schonbrun, E.; Yang, T.; Crozier, K. B. Experimental Observation of Narrow Surface Plasmon Resonances in Gold Nanoparticle Arrays. Appl. Phys. Lett. 2008, 93, 181108.

(37) Auguié, B.; Barnes, W. Collective Resonances in Gold Nanoparticle Arrays. Phys. Rev. Lett. 2008, 101, 143902.

(38) Vecchi, G.; Giannini, V.; Gómez Rivas, J. Surface Modes in Plasmonic Crystals Induced by Diffractive Coupling of Nanoantennas. Phys. Rev. B 2009, 80, 201401.

(39) Nikitin, A. G.; Kabashin, A. V.; Dallaporta, H. Plasmonic Resonances in Diffractive Arrays of Gold Nanoantennas: Near and Far Field Effects. Opt. Express 2012, 20, 27941-27952.
(40) Kuznetsov, A. I.; Evlyukhin, A. B.; Gonçalves, M. R.; Reinhardt, C.; Koroleva, A.; Arnedillo, M. L.; Kiyan, R.; Marti, O.; Chichkov, B. N. Laser Fabrication of Large-Scale Nanoparticle Arrays for Sensing Applications. ACS Nano 2011, 5, 4843-4849.

(41) Offermans, P.; Schaafsma, M. C.; Rodriguez, S. R. K.; Zhang, Y.; Crego-Calama, M.; Brongersma, S. H.; Gómez Rivas, J. Universal Scaling of the Figure of Merit of Plasmonic Sensors. ACS Nano 2011, 5, 5151-5157.

(42) Auguié, B.; Bendaña, X. M.; Barnes, W. L.; García de Abajo, F. J. Diffractive Arrays of Gold Nanoparticles near an Interface: Critical Role of the Substrate. Phys. Rev. B 2010, 82, 155447.

(43) Jung, L.; Campbell, C.; Chinowsky, T. Quantitative Interpretation of the Response of Surface Plasmon Resonance Sensors to Adsorbed Films. Langmuir 1998, 7463, 5636-5648.

(44) Jonsson, M. P.; Jönsson, P.; Höök, F. Simultaneous Nanoplasmonic and Quartz Crystal Microbalance Sensing: Analysis of Biomolecular Conformational Changes and Quantification of the Bound Molecular Mass. Anal. Chem. 2008, 80, 7988-7995.

(45) Zijlstra, P.; Paulo, P. M. R.; Orrit, M. Optical Detection of Single Non-Absorbing Molecules Using the Surface Plasmon Resonance of a Gold Nanorod. Nat. Nanotechnol. 2012, 7, 379-382.

(46) Aćimović, S. S.; Kreuzer, M. P.; González, M. U.; Quidant, R. Plasmon near-Field Coupling in Metal Dimers as a Step toward SingleMolecule Sensing. ACS Nano 2009, 3, 1231-1237.

(47) White, I. M.; Fan, X. On the Performance Quantification of Resonant Refractive Index Sensors. Opt. Express 2008, 16, 1020-1028.

(48) Dahlin, A. B.; Tegenfeldt, J. O.; Höök, F. Improving the Instrumental Resolution of Sensors Based on Localized Surface Plasmon Resonance. Anal. Chem. 2006, 78, 4416-4423.

(49) Nusz, G. J.; Marinakos, S. M.; Curry, A. C.; Dahlin, A.; Höök, F.; Wax, A.; Chilkoti, A. Label-Free Plasmonic Detection of Biomolecular Binding by a Single Gold Nanorod. Anal. Chem. 2008, 80, 984-989.

(50) Seo, E. K.; Lee, J. W.; Sung-Suh, H. M.; Sung, M. M. Atomic Layer Deposition of Titanium Oxide on Self-Assembled-MonolayerCoated Gold. Chem. Mater. 2004, 16, 1878-1883. 